\title{
Some Common Fixed Point Theorems Satisfying Meir-Keeler Type Contractive Conditions
}

\author{
Dinesh Panthi \\ Department of Mathematics, Valmeeki Campus, Nepal Sanskrit University, Kathmandu, Nepal \\ Email: panthid06@gmail.com
}

How to cite this paper: Panthi, D. (2018)

Some Common Fixed Point Theorems Satisfying Meir-Keeler Type Contractive Conditions. Open Journal of Discrete Mathematics, 8, 35-47.

https://doi.org/10.4236/ojdm.2018.82004

Received: February 20, 2018

Accepted: April 17, 2018

Published: April 20, 2018

Copyright $\odot 2018$ by author and Scientific Research Publishing Inc. This work is licensed under the Creative Commons Attribution International License (CC BY 4.0).

http://creativecommons.org/licenses/by/4.0/

\section{cc) (i) Open Access}

\begin{abstract}
In this article, we establish some common fixed point results for two pairs of compatible mappings satisfying Meir-Keeler type contractive conditions in metric space and dislocated metric space which extend and improve some similar fixed point results in the literature.

\section{Keywords}

Common Fixed Point, Compatible Maps, Cauchy Sequence, Continuous Function
\end{abstract}

\section{Introduction}

The word metrics plays a dominant role in most of the fields like, geometry, economics, statistics, graph theory, probability theory, coding theory, game theory, pattern recognition, computer graphics, theory of information and computer semantics, molecular biology etc. M Frechet in 1906, first time introduced the notion of metric space which is now a very useful topic in mathematical analysis. In 1912, L. E. J. Brouwer [1] established a topological fixed point theorem. In 1922, S. Banach [2] established a contraction mapping theorem in a complete metric space, is a primary result of functional analysis. After the establishment of contraction mapping theorem, various authors generalized the theorem and established a huge number of fixed point results in the literature.

In 1969, A. Meir and E. Keeler [3] obtained a remarkable generalization of Banach Contraction principle with the notion of weakly uniformly strict contraction which is famous as $(\epsilon-\delta)$ contraction principle. This theorem has also been generalized by various authors for single, pairs and even for sequence of mappings.

There exists a vast literature which generalizes the result of Meir and Keeler. Maiti and Pal [4] established a fixed point theorem for a self map $\mathrm{T}$ of a metric 
space satisfying the following condition which is the generalization of weakly uniformly strict contraction (1). For every $\varepsilon>0$ there exists a $\delta>0$ such that

$$
\varepsilon \leq \max \{d(x, y), d(x, T y), d(y, T y)\}<\varepsilon+\delta \Rightarrow d(T x, T y)<\varepsilon
$$

Park-Rhoades [5] and Rao-Rao [6] extended this result for two self mapping $S$ and $T$ in a metric space $(X, d)$ satisfying the condition

$$
\begin{aligned}
& \varepsilon \leq \max \left\{d(S x, S y), d(S x, T x), d(S y, T y), \frac{1}{2}[d(S x, T y)+d(S y, T x)]\right\}<\varepsilon+\delta \\
& \Rightarrow d(T x, T y)<\varepsilon
\end{aligned}
$$

In 1986, Jungck [7] and Pant [8] extended the results for four mappings.

The study of common fixed points satisfying contractive type conditions has been a very active field of research activity. The most general common fixed point theorems for four mappings, say $A, B, S$ and $T$ of a metric space $(X, d)$ use either a.

Banach type contractive condition of the form

1) $d(A x, B y) \leq h M(x, y), \quad 0 \leq h<1$

where

$$
M(x, y)=\max \left\{d(S x, T y), d(A x, S x), d(B y, T y), \frac{1}{2}[d(A x, T y)+d(B y, S x)]\right\}
$$

a Meir-Keeler type contractive definition

2) given $\epsilon>0$ there exists a $\delta>0$ such that

$$
\epsilon \leq M(x, y)<\epsilon+\delta d(A x, B y)<\epsilon
$$

or $\phi$-contractive condition of the form

3) $d(A x, B y) \leq \phi(M(x, y))$

where $\phi: \mathbb{R}_{+} \rightarrow \mathbb{R}_{+}$is such that $\phi(t)<t$ for each $t>0$

The contractive condition (2) ensures the existence of a fixed point, only when $\phi$ satisfies some additional conditions.

The following conditions on the function $\phi$ have introduced and employed by various authors for the establishment of fixed point

1) $\phi(t)$ is non decreasing and $\frac{t}{t-\phi(t)}$ is non increasing [9].

2) $\phi(t)$ is non decreasing and $\lim _{n \rightarrow \infty} \phi^{n}(t)=0$ for each $t>0$ [10] [11].

3) $\phi$ is upper semi-continuous [11] [12] [13].

4) $\phi$ is non decreasing and continuous from the right [14].

In 1985, S. G. Matthwes [15] generalized Banach Contraction Mapping Theorem under metric domains in domain theory. In 2000, P. Hitzeler and A. K. Seda [16] provided a generalization on the notion of topology and gave a name as dislocated topology. He presented variants of Banach Contraction Principle for various modified forms of metric space including dislocated metric space. Since then, many authors have established fixed point theorems in dislocated metric space. In the literature one can find many interesting recent articles in the field of dislocated metric space (See for examples [17] [18] [19] [20] [21]). The 
study of dislocated metric plays very important role in Topology, semantics of logic programming and in electronics engineering.

In this paper, we establish some common fixed point theorems for two pairs of compatible and weakly compatible mappings satisfying Meir-Keeler type contractive condition in dislocated metric space.

\section{Preliminaries}

We start with the following definitions and theorems.

Definition 1. [16] Let $X$ be a non empty set and let $d: X \times X \rightarrow[0, \infty)$ be a function satisfying the following conditions.

1) $d(x, x)=0$

2) $d(x, y)=0$ implies $x=y$.

3) $d(x, y)=d(y, x)$

4) $d(x, y) \leq d(x, z)+d(z, y)$ for all $x, y, z \in X$.

If $d$ satisfies the conditions $1-4$ Then $d$ is called the metric on $X$ and the pair $(X, d)$ is called the metric space. If $d$ satisfies the conditions $2-4$, then $d$ is called the $d$-metric on $X$ and the pair $(X, d)$ is called the dislocated metric space.

Definition 2. [3] A self mapping $T$ of a metric space $(X, d)$ is called a weakly uniformly strict contraction or simply an $(\varepsilon-\delta)$ contraction if for each $\varepsilon>0$ there exists $\delta>0$ such that for all $x, y \in X$

$$
\varepsilon \leq d(x, y)<\varepsilon+\delta \Rightarrow d(T x, T y)<\varepsilon
$$

Theorem 1. [3] Let $(X, d)$ be a complete metric space and $T: X \rightarrow X$ is weakly uniformly strict contraction then $T$ has a unique common fixed point, say $z$ and for any $x \in X, \lim _{n \rightarrow \infty} T^{n} x=z$.

Definition 3. [7] Two mappings $S$ and T from a metric space $(X, d)$ into itself are called compatible if

$$
\lim _{n \rightarrow \infty} d\left(S T x_{n}, T S x_{n}\right)=0
$$

whenever $\left\{x_{n}\right\}$ is a sequence in $X$ such that $\lim _{n \rightarrow \infty} S x_{n}=\lim _{n \rightarrow \infty} T x_{n}=x$ for some $x \in X$

Definition 4. [22] Let $A$ and $S$ be mappings from a metric space $(X, d)$ into itself. Then, $A$ and $S$ are said to be weakly compatible if they commute at their coincident point, that is, $A x=S x$ for some $x \in X$ implies $A S x=S A x$.

Definition 5. [23] Two self mappings $A$ and $S$ of a metric space $(X, d)$ are called reciprocally continuous if $\lim _{n} A S x_{n}=A t$ and $\lim _{n} S A x_{n}=S t$ whenever $\left\{x_{n}\right\}$ is a sequence such that $\lim _{n} A x_{n}=\lim _{n} S x_{n}=t$ for some $t \in X$.

If $A$ and $S$ both are continuous they are obviously reciprocally continuous but the converse is not true.

\section{Main Results}

Now we establish the following lemma in dislocated metric space.

Lemma 1. Let $(X, d)$ be a dislocated metric space. Let $A, B, S, T: X \rightarrow X$ be mappings satisfying the conditions 


$$
A(X) \subseteq T(X) \text { and } B(X) \subseteq S(X)
$$

Assume further that given for each $\varepsilon>0$ there exists $\delta>0$ such that for all $x, y \in X$

$$
\varepsilon \leq M(x, y)<\varepsilon+\delta \Rightarrow d(A x, B y) \leq \varepsilon
$$

and

$$
d(A x, B y)<M(x, y) \text { whenever } M(x, y)>0
$$

where

$$
M(x, y)=\max \left\{d(S x, T y), d(A x, S x), d(B y, T y), \frac{1}{2} d(S x, B y), \frac{1}{2} d(A x, T y)\right\}
$$

then for each $x_{0} \in X$, the sequence $\left\{y_{n}\right\}$ in $X$ defined by the rule

$$
y_{2 n}=A x_{2 n}=T x_{2 n+1}, \quad y_{2 n+1}=B x_{2 n+1}=S x_{2 n+2}
$$

is a Cauchy sequence.

Proof. let $x_{0} \in X$, then by condition (2) we can define a sequence $\left\{A x_{0}, B x_{1}, A x_{2}, B x_{3}, A x_{2 n}, B x_{2 n+1}\right\}$ such that

$$
y_{2 n}=A x_{2 n}=T x_{2 n+1} \text { and } y_{2 n+1}=B x_{2 n+1}=S x_{2 n+2} \text { for } n \in \mathbb{N} \bigcup\{0\}
$$

Now we have

$$
\begin{aligned}
d & \left(y_{2 n+1}, y_{2 n+2}\right)=d\left(A x_{2 n+1}, B x_{2 n+2}\right) \\
< & \max \left\{d\left(S x_{2 n+1}, T x_{2 n+2}\right), d\left(A x_{2 n+1}, S x_{2 n+1}\right), d\left(B x_{2 n+2}, T x_{2 n+2}\right),\right. \\
& \left.\frac{1}{2} d\left(S x_{2 n+1}, B x_{2 n+2}\right), \frac{1}{2} d\left(A x_{2 n+1}, T x_{2 n+2}\right)\right\} \\
= & \max \left\{d\left(y_{2 n}, y_{2 n+1}\right), d\left(y_{2 n+1}, y_{2 n}\right), d\left(y_{2 n+2}, y_{2 n+1}\right),\right. \\
& \left.\frac{1}{2} d\left(y_{2 n}, y_{2 n+2}\right), \frac{1}{2} d\left(y_{2 n+1}, y_{2 n+1}\right)\right\}
\end{aligned}
$$

But,

$$
\begin{aligned}
\frac{1}{2} d\left(y_{2 n}, y_{2 n+2}\right) & \leq \frac{1}{2}\left[d\left(y_{2 n}, y_{2 n+1}\right)+d\left(y_{2 n+1}, y_{2 n+2}\right)\right] \\
& \leq \max \left\{d\left(y_{2 n}, y_{2 n+1}\right), d\left(y_{2 n+1}, y_{2 n+2}\right)\right\}
\end{aligned}
$$

and,

$$
\begin{aligned}
\frac{1}{2} d\left(y_{2 n+1}, y_{2 n+1}\right) & \leq \frac{1}{2}\left[d\left(y_{2 n+1}, y_{2 n}\right)+d\left(y_{2 n}, y_{2 n+1}\right)\right] \\
& \leq \max \left\{d\left(y_{2 n+1}, y_{2 n}\right), d\left(y_{2 n+1}, y_{2 n}\right)\right\} \\
& =d\left(y_{2 n+1}, y_{2 n}\right)
\end{aligned}
$$

Hence, $d\left(y_{2 n+1}, y_{2 n+2}\right)<\max \left\{d\left(y_{2 n}, y_{2 n+1}\right), d\left(y_{2 n+2}, y_{2 n+1}\right)\right\}$ which implies that, $d\left(y_{2 n+1}, y_{2 n+2}\right)<d\left(y_{2 n}, y_{2 n+1}\right)$

[ $d\left(y_{2 n+1}, y_{2 n+2}\right)<d\left(y_{2 n+1}, y_{2 n+2}\right)$ is impossible.]

Similarly, we can show that

$$
d\left(y_{2 n+1}, y_{2 n}\right)<d\left(y_{2 n}, y_{2 n-1}\right)
$$


Consequently, we conclude that $d\left(y_{n+1}, y_{n}\right)<d\left(y_{n}, y_{n-1}\right)$. For $n$ even or odd, the last inequality implies that $\left\{y_{n}\right\}$ is a Cauchy sequence.

Now, we establish a common fixed point theorem for two pairs of compatible mappings in metric space.

Theorem 2. Let $(X, d)$ be a complete metric space. Let $A, B, S, T: X \rightarrow X$ such that the pairs $(A, S)$ and $(B, T)$ be compatible mappings which satisfy the following conditions

$$
A(X) \subset T(X), \quad B(X) \subset S(X)
$$

Given, $\varepsilon>0, \exists \delta>0$, such that

$$
\varepsilon<M(x, y)<\epsilon+\delta \Rightarrow d(A x, B y) \leq \varepsilon
$$

where

$$
\begin{gathered}
M(x, y)=\max \left\{d(S x, T y), d(A x, S x), d(B y, T y), \frac{1}{2}[d(A x, T y)+d(B y, S x)]\right\} \\
d(A x, B y)<k\left\{\frac{d(S x, T y) d(S x, A x)}{d(S x, B y)}+d(S x, T y)+d(A x, S x)+d(B y, T y)\right. \\
\left.+d(A x, T y)+d(B y, S x)+\frac{d(B y, T y) d(A x, T y)}{d(S x, B y)}\right\}
\end{gathered}
$$

$0<k \leq \frac{1}{3}$. Suppose that the mappings in one of the pairs $(A, S)$ or $(B, T)$ are reciprocally continuous, then $A, B, S$ and Thave a unique common fixed point.

\section{Proof:}

let $x_{0} \in X$ be any point in $X$. Define sequences $\left\{x_{n}\right\}$ and $\left\{y_{n}\right\}$ in $X$ given by the rule

$$
y_{2 n}=A x_{2 n}=T x_{2 n+1} \text { and } y_{2 n+1}=B x_{2 n+1}=S x_{2 n+2} \text { for } n \in \mathbb{N} \bigcup\{0\}
$$

then by Jachymski's lemma [11], $\left\{y_{n}\right\}$ is a Cauchy sequence. Sine $X$ is complete, there exists a point $z \in X$ such that $y_{n} \rightarrow z$. Also the sequences

$$
y_{2 n}=A x_{2 n}=T x_{2 n+1} \rightarrow z \text { and } y_{2 n}=A x_{2 n}=T x_{2 n+1} \rightarrow z
$$

Suppose that the pair $(A, S)$ is reciprocally continuous, then $A S x_{n} \rightarrow A z$ and $S A x_{n} \rightarrow S z$. Since the pair $(A, S)$ is compatible so

$$
\lim _{n \rightarrow \infty} S x_{2 n}=\lim _{n \rightarrow \infty} A x_{2 n}=Z \text { and } \lim _{n \rightarrow \infty} S x_{2 n}=\lim _{n \rightarrow \infty} A x_{2 n}=z
$$

This implies that $d(A z, S z)=0$. Hence $A z=S z$.

Since, $A(X) \subset T(X)$ there exists a point $w \in X$ such that $A z=T w$. We claim that $B w=T w$. If $B w \neq T w$, then by using condition (8) we get,

$$
\begin{aligned}
d(A z, B w)< & k\left\{\frac{d(S z, T w) d(S z, A z)}{d(S z, B w)}+d(S z, T w)+d(A z, S z)+d(B w, T w)\right. \\
& \left.+d(A z, T w)+d(B w, S z)+\frac{d(B w, T w) d(A z, T w)}{d(S z, B w)}\right\} \\
= & k\{0+0+0+d(B w, A z)+0+d(B w, A z)+0\} \\
= & 2 k d(A z, B w)
\end{aligned}
$$


which is a contradiction. So $d(A z, B w)=0 A z=B w$. Hence $A z=S z=T w=B w$.

Since the compatible maps commute at their coincidence point, we get $A S z=S A z$. This further implies that $A A z=A S z=S A z=S S z$. If $A z \neq A A z$, by condition (8) we get

$$
\begin{aligned}
& d(A z, A A z)=d(B w, A A z)=d(A A z, B w) \\
& <k\left(\frac{d(S A z, T w) d(S A z, A A z)}{d(S A z, B w)}+d(S A z, T w)+d(A A z, S A z)\right. \\
& \left.+d(B w, T w)+d(A A z, T w)+d(B w, S A z)+\frac{d(B w, T w) d(A A z, T w)}{d(S A z, B w)}\right\} \\
& =k\{0+d(A A z, B w)+0+0+d(A A z, B w)+d(B w, A A z)+0\} \\
& =3 k d(A A z, B w)=3 k d(A A z, A z)
\end{aligned}
$$

which is a contradictions, so $d(A z, A A z)=0 \Rightarrow A z=A A z$. Hence, $A z=A A z=S A z$. Thus $A z$ is the common fixed point of the mappings $A$ and $S$. Similarly we obtain $B w(=A z)$ is the common fixed point of the mappings $B$ and $T$.

\section{Uniqueness:}

If possible, let $u$ and $v(u \neq v)$ are two common fixed points of the maps $A$, $B, S$ and $T$. Now by virtu of (8)

$$
\begin{aligned}
& d(u, v)=d(A u, B v)<k\left\{\frac{d(S u, T v) d(S u, A u)}{d(S u, B v)}+d(S u, T v)+d(A u, S u)\right. \\
& \left.+d(B v, T v)+d(A u, T v)+d(B v, S u)+\frac{d(B v, T v) d(A u, T v)}{d(S u, B v)}\right\} \\
& =k\left\{\frac{d(u, v) d(u, u)}{d(u, v)}+d(u, v)+d(u, u)+d(v, v)\right. \\
& \left.+d(u, v)+d(v, u)+\frac{d(v, v) d(u, v)}{d(u, v)}\right\} \\
& =3 k d(u, v)
\end{aligned}
$$

which is a contradiction. This shows that $d(u, v)=0 \Rightarrow u=v$

The proof is similar when the mappings $B$ and $T$ are assumed compatible and reciprocally continuous. This completes the proof of the theorem.

Now, on the light of above theorem, one can establish the following corollaries easily.

Corrollary 1. Let $(X, d)$ be a complete metric space. Let $A, B, S: X \rightarrow X$ such that the pairs $(A, S)$ and $(B, S)$ be compatible mappings which satisfy the following conditions

$$
A(X), B(X) \subset S(X)
$$

Given, $\varepsilon>0, \exists \delta>0$, such that

$$
\varepsilon<M(x, y)<\epsilon+\delta \Rightarrow d(A x, B y) \leq \varepsilon
$$

where 


$$
\begin{gathered}
M(x, y)=\max \left\{d(S x, S y), d(A x, S x), d(B y, S y), \frac{1}{2}[d(A x, S y)+d(B y, S x)]\right\} \\
d(A x, B y)<k\left\{\frac{d(S x, S y) d(S x, A x)}{d(S x, B y)}+d(S x, S y)+d(A x, S x)+d(B y, S y)\right. \\
\left.+d(A x, S y)+d(B y, S x)+\frac{d(B y, S y) d(A x, S y)}{d(S x, B y)}\right\}
\end{gathered}
$$

$0<k \leq \frac{1}{3}$ suppose that the mappings in one of the pairs $(A, S)$ or $(B, S)$ are reciprocally continuous, then $A, B$ and $S$ have a unique common fixed point.

Corrollary 2. Let $(X, d)$ be a complete metric space. Let $A, S, T: X \rightarrow X$ such that the pairs $(A, S)$ and $(A, T)$ be compatible mappings which satisfy the following conditions

$$
A(X) \subset T(X), \quad A(X) \subset S(X)
$$

Given, $\varepsilon>0, \exists \delta>0$, such that

$$
\varepsilon<M(x, y)<\epsilon+\delta \Rightarrow d(A x, A y) \leq \varepsilon
$$

where

$$
\begin{gathered}
M(x, y)=\max \left\{d(S x, T y), d(A x, S x), d(A y, T y), \frac{1}{2}[d(A x, T y)+d(A y, S x)]\right\} \\
d(A x, A y)<k\left\{\frac{d(S x, T y) d(S x, A x)}{d(S x, A y)}+d(S x, T y)+d(A x, S x)+d(A y, T y)\right. \\
\left.+d(A x, T y)+d(A y, S x)+\frac{d(A y, T y) d(A x, T y)}{d(S x, A y)}\right\}
\end{gathered}
$$

$0<k \leq \frac{1}{3}$. Suppose that the mappings in one of the pairs $(A, S)$ or $(A, T)$ are reciprocally continuous, then $A, S$ and $T$ have a unique common fixed point.

Corrollary 3. Let $(X, d)$ be a complete metric space. Let $A, S: X \rightarrow X$ such that the pair $(A, S)$ be compatible mappings which satisfy the following conditions

$$
A(X) \subset S(X)
$$

Given, $\varepsilon>0, \exists \delta>0$, such that

$$
\varepsilon<M(x, y)<\epsilon+\delta \Rightarrow d(A x, A y) \leq \varepsilon
$$

where

$$
\begin{gathered}
M(x, y)=\max \left\{d(S x, S y), d(A x, S x), d(A y, S y), \frac{1}{2}[d(A x, S y)+d(A y, S x)]\right\} \\
d(A x, A y)<k\left\{\frac{d(S x, S y) d(S x, A x)}{d(S x, A y)}+d(S x, S y)+d(A x, S x)+d(A y, S y)\right. \\
\left.+d(A x, S y)+d(A y, S x)+\frac{d(A y, S y) d(A x, S y)}{d(S x, A y)}\right\}
\end{gathered}
$$


$0<k \leq \frac{1}{3}$. Suppose that the pair $(A, S)$ is reciprocally continuous, then $A$, and $S$ have a unique common fixed point.

Corrollary 4. Let $(X, d)$ be a complete metric space. Let $A, B, I_{X}: X \rightarrow X$ such that the pairs $\left(A, I_{X}\right)$ and $\left(B, I_{X}\right)$ be compatible mappings which satisfy the following conditions

$$
A(X), B(X) \subset I_{X}
$$

Given, $\varepsilon>0, \exists \delta>0$, such that

$$
\varepsilon<M(x, y)<\epsilon+\delta \Rightarrow d(A x, B y) \leq \varepsilon
$$

where

$$
\begin{gathered}
M(x, y)=\max \left\{d(x, y), d(A x, x), d(B y, y), \frac{1}{2}[d(A x, y)+d(B y, x)]\right\} \\
d(A x, B y)<k\left\{\frac{d(x, y) d(x, A x)}{d(x, B y)}+d(x, y)+d(A x, x)+d(B y, y)\right. \\
\left.+d(A x, y)+d(B y, x)+\frac{d(B y, y) d(A x, y)}{d(x, B y)}\right\}
\end{gathered}
$$

$0<k \leq \frac{1}{3}$. Suppose that the mappings in one of the pairs $\left(A, I_{X}\right)$ or $\left(B, I_{X}\right)$ are reciprocally continuous, then $A, B, I_{X}$ have a unique common fixed point.

Now, we establish a common fixed point theorem for two pais of compatible mappings in dislocated metric space.

Theorem 3. Let $(X, d)$ be a complete dislocated metric space. Let $A, B, S, T: X \rightarrow X$ such that the pairs $(A, S)$ and $(B, T)$ be compatible mappings which satisfy the following conditions

$$
A(X) \subset T(X), \quad B(X) \subset S(X)
$$

Given, $\varepsilon>0, \exists \delta>0$, such that

$$
\varepsilon<M(x, y)<\epsilon+\delta \Rightarrow d(A x, B y) \leq \varepsilon
$$

where

$$
\begin{aligned}
& M(x, y)=\max \left\{d(S x, T y), d(A x, S x), d(B y, T y), \frac{1}{2} d(A x, T y), \frac{1}{2} d(B y, S x)\right\} \\
& d(A x, B y)<\phi(k\{d(S x, T y)+d(A x, S x)+d(B y, T y)+d(A x, T y)+d(B y, S x)\})
\end{aligned}
$$

where, $0<k \leq \frac{1}{8}$ and $\phi: \mathbb{R}_{+} \rightarrow \mathbb{R}_{+}$is such that $\phi(t)<t$ for each $t>0$. If one of the mappings $A, B, S$ or $T$ be continuous then $A, B, S$ and $T$ have a unique common fixed point.

Proof. Let $x_{0} \in X$ be any point in $X$. Define sequences $\left\{x_{n}\right\}$ and $\left\{y_{n}\right\}$ in $X$ given by the rule

$$
y_{2 n}=A x_{2 n}=T x_{2 n+1} \text { and } y_{2 n+1}=B x_{2 n+1}=S x_{2 n+2} \text { for } n \in \mathbb{R} \bigcup\{0\}
$$


then by above lemma (1), $\left\{y_{n}\right\}$ is a Cauchy sequence. Sine $X$ is complete there exists a point $z \in X$ such that $y_{n} \rightarrow z$. Also the sequences

$$
y_{2 n}=A x_{2 n}=T x_{2 n+1} \rightarrow z \text { and } y_{2 n+1}=B x_{2 n+1}=S x_{2 n+2} \rightarrow z
$$

Suppose that $S$ is continuous, then $S S x_{2 n} \rightarrow S z$ and $S A x_{2 n} \rightarrow S z$. Since the pair $(A, S)$ is compatible so

$$
A S x_{2 n} \rightarrow S z
$$

Since $A(X) \subset T(X)$, corresponding to each value of $n$, there exists a sequence $z_{2 n} \in X$ such that $A S x_{2 n}=T z_{2 n}$.

Thus $A S x_{2 n}=T z_{2 n} \rightarrow S z$ and $S S x_{2 n} \rightarrow S z$.

We assert that

$$
\lim _{n} B z_{2 n}=S z
$$

If not, there exists a subsequence $B z_{2 m}$ of $B z_{2 n}$, a number $r>0$ and a positive number $N$ such that for each $m \geq N$, we have

$$
d\left(A S x_{2 m}, B z_{2 m}\right) \geq r \text { and } d\left(B z_{2 m}, S z\right) \geq r
$$

Moreover, by virtu of (14) for large $m$ we obtain,

$$
\begin{aligned}
d\left(A S x_{2 m}, B z_{2 m}\right)< & \phi\left(k \left\{d\left(S S x_{2 m}, T z_{2 m}\right)+d\left(A S x_{2 m}, S S x_{2 m}\right)+d\left(B z_{2 m}, T z_{2 m}\right)\right.\right. \\
& \left.\left.+d\left(A S x_{2 m}, T z_{2 m}\right)+d\left(B z_{2 m}, S S x_{2 m}\right)\right\}\right)
\end{aligned}
$$

now taking limit as $m \rightarrow \infty$ we have

$$
\begin{gathered}
\lim _{m} d\left(S z, B z_{2 m}\right)<\lim _{m} \phi\left(k\left\{d\left(B z_{2 m}, S z\right)+d\left(B z_{2 m}, S z\right)\right\}\right) \\
\lim _{m} d\left(S z, B z_{2 m}\right)<\lim _{m} \phi\left(2 k d\left(B z_{2 m}, S z\right)\right) \\
\lim _{m} d\left(S z, B z_{2 m}\right)<2 k \lim _{m} d\left(B z_{2 m}, S z\right)
\end{gathered}
$$

which is a contradiction, Hence

$$
\lim _{m} d\left(S z, B z_{2 m}\right)=0
$$

This represents that,

$$
\lim _{m} B z_{2 n}=S z
$$

We claim that $A z=S z$

If $A z \neq S z$, then by virtu of (14) for large $n$, We obtain

$$
\begin{aligned}
d\left(A z, B z_{2 n}\right)< & \phi\left(k \left\{d\left(S z, T z_{2 n}\right)+d(A z, S z)+d\left(B z_{2 n}, T z_{2 n}\right)\right.\right. \\
& \left.\left.+d\left(A z, T z_{2 n}\right)+d\left(B z_{2 n}, S z\right)\right\}\right)
\end{aligned}
$$

Now letting $n \rightarrow \infty$ we obtain,

$$
\begin{aligned}
d(A z, S z) & <\phi(k\{d(S z, S z)+d(A z, S z)+d(S z, S z)+d(A z, S z)+d(S z, S z)\}) \\
& \leq \phi(k 8 d(A z, S z))<8 k d(A z, S z)
\end{aligned}
$$

which is a contradiction. Therefore, $d(A z, S z)=0 \Rightarrow A z=S z$.

Since, $A(X) \subset T(X)$ there exists a point $w \in X$ such that $A z=T w$. We 
claim that $B w=T w$. If $B w \neq T w$ by using condition (14) we get,

$$
\begin{aligned}
& d(A z, B w) \\
& <\phi(k\{d(S z, T w)+d(A z, S z)+d(B w, T w)+d(A z, T w)+d(B w, S z)\}) \\
& =\phi(k\{d(A z, A z)+d(A z, A z)+d(B w, A z)+d(A z, A z)+d(B w, A z)\}) \\
& =\phi(k\{3 d(A z, A z)+2 d(A z, B w)\}) \\
& <8 k d(A z, B w)
\end{aligned}
$$

which is a contradiction. So $d(A z, B w)=0 \Rightarrow A z=B w$. Hence $A z=S z=T w=B w$.

Sice the compatible maps commute at their coincidence point, we get $A S z=S A z$. This further implies that $A A z=A S z=S A z=S S z$.

If $A z \neq A A z$, by condition (14) we get

$$
\begin{aligned}
& d(A z, A A z)=d(B w, A A z)=d(A A z, B w) \\
& <\phi(k\{d(S A z, T w)+d(A A z, S A z)+d(B w, T w)+d(A A z, T w)+d(B w, S A z)\}) \\
& =\phi(k\{d(A A z, A z)+d(A A z, A A z)+d(A z, A z)+d(A A z, A z)+d(A z, A A z)\}) \\
& =\phi(k\{3 d(A A z, A z)+d(A A z, A A z)+d(A z, A z)\}) \\
& \leq \phi(7 k d(A A z, A z))<7 k d(A A z, A z)
\end{aligned}
$$

which is a contradictions, so $d(A z, A A z)=0 \Rightarrow A z=A A z$. Hence, $A z=A A z=S A z$. Thus $A z$ is the common fixed point of the mappings $A$ and $S$.

Similarly we obtain $B w(=A z)$ is the common fixed point of the mappings $B$ and $T$ when $T$ is supposed to be continuous.

\section{Uniqueness:}

Let $u$ and $v(u \neq v)$ are two common fixed points of the mappings $A, B, S$ and $T$. Now by virtu of (14),

$$
\begin{aligned}
& d(u, v)=d(A u, B v) \\
& <\phi(k\{d(S u, T v)+d(A u, S u)+d(B v, T v)+d(A u, T v)+d(B v, S u)\}) \\
& =\phi(k\{d(u, v)+d(u, u)+d(v, v)+d(u, v)+d(v, u)\}) \\
& =\phi(k\{3 d(u, v)+d(u, u)+d(v, v)\}) \\
& \leq \phi(7 k d(u, v))<7 k d(u, v)
\end{aligned}
$$

which is a contradiction. Hence $d(u, v)=0 \Rightarrow u=v$.

Moreover, the proof follows on similar lines when $A$ or $B$ is assumed to be continuous since $A(X) \subset T(X)$ and $B(X) \subset S(X)$.

This completes the proof of the theorem.

Now, in the light of the above theorem one can establish the following corollaries easily.

Corrollary 5. Let $(X, d)$ be a complete dislocated metric space. Let $A, B, S: X \rightarrow X$ such that the pairs $(A, S)$ and $(B, S)$ be compatible mappings which satisfy the following conditions

$$
A(X) \text { and } B(X) \subset S(X)
$$


Given, $\varepsilon>0, \exists \delta>0$, such that

$$
\varepsilon<M(x, y)<\epsilon+\delta \Rightarrow d(A x, B y) \leq \varepsilon
$$

where

$$
\begin{aligned}
& M(x, y)=\max \left\{d(S x, S y), d(A x, S x), d(B y, S y), \frac{1}{2} d(A x, S y), \frac{1}{2} d(B y, S x)\right\} \\
& d(A x, B y)<\phi(k\{d(S x, S y)+d(A x, S x)+d(B y, S y)+d(A x, S y)+d(B y, S x)\})
\end{aligned}
$$

where, $0<k \leq \frac{1}{8}$ and $\phi: \mathbb{R}_{+} \rightarrow \mathbb{R}_{+}$is such that $\phi(t)<t$ for each $t>0$. If one of the mappings $A, B$ or $S$ be continuous then $A, B$ and $S$ have a unique common fixed point.

Corrollary 6. Let $(X, d)$ be a complete dislocated metric space. Let $A, S, T: X \rightarrow X$ such that the pairs $(A, S)$ and $(A, T)$ be compatible mappings which satisfy the following conditions

$$
A(X) \subset T(X) \text { and } S(X)
$$

Given, $\varepsilon>0, \exists \delta>0$, such that

$$
\varepsilon<M(x, y)<\epsilon+\delta \Rightarrow d(A x, A y) \leq \varepsilon
$$

where

$$
\begin{aligned}
& M(x, y)=\max \left\{d(S x, T y), d(A x, S x), d(A y, T y), \frac{1}{2} d(A x, T y), \frac{1}{2} d(A y, S x)\right\} \\
& d(A x, A y)<\phi(k\{d(S x, T y)+d(A x, S x)+d(A y, T y)+d(A x, T y)+d(A y, S x)\})
\end{aligned}
$$

where, $0<k \leq \frac{1}{8}$ and $\phi: \mathbb{R}_{+} \rightarrow \mathbb{R}_{+}$is such that $\phi(t)<t$ for each $t>0$. If one of the mappings $A, S$ or $T$ be continuous then $A, S$ and $T$ have a unique common fixed point.

Corrollary 7. Let $(X, d)$ be a complete dislocated metric space. Let $A, S: X \rightarrow X$ such that the pair $(A, S)$ be compatible mappings which satisfy the following conditions

$$
A(X) \subset S(X)
$$

Given, $\varepsilon>0, \exists \delta>0$, such that

$$
\varepsilon<M(x, y)<\epsilon+\delta \Rightarrow d(A x, B y) \leq \varepsilon
$$

where

$$
\begin{aligned}
& M(x, y)=\max \left\{d(S x, S y), d(A x, S x), d(A y, T y), \frac{1}{2} d(A x, S y), \frac{1}{2} d(A y, S x)\right\} \\
& d(A x, A y)<\phi(k\{d(S x, S y)+d(A x, S x)+d(A y, S y)+d(A x, S y)+d(A y, S x)\})
\end{aligned}
$$

where, $0<k \leq \frac{1}{8}$ and $\phi: \mathbb{R}_{+} \rightarrow \mathbb{R}_{+}$is such that $\phi(t)<t$ for each $t>0$. If one of the mappings $A$ or $S$ be continuous then $A$ and $S$ have a unique common fixed point. 
Corrollary 8. Let $(X, d)$ be a complete dislocated metric space. Let $A, B, I_{X}: X \rightarrow X$ such that the pairs $\left(A, I_{X}\right)$ and $\left(B, I_{X}\right)$ be compatible mappings which satisfy the following conditions

$$
A(X) \text { and } B(X) \subset I_{X}
$$

Given, $\varepsilon>0, \exists \delta>0$, such that

$$
\varepsilon<M(x, y)<\epsilon+\delta \Rightarrow d(A x, B y) \leq \varepsilon
$$

where

$$
\begin{aligned}
& M(x, y)=\max \left\{d(x, y), d(A x, x), d(B y, y), \frac{1}{2} d(A x, y), \frac{1}{2} d(B y, x)\right\} \\
& d(A x, B y)<\phi(k\{d(x, y)+d(A x, x)+d(B y, y)+d(A x, y)+d(B y, x)\})
\end{aligned}
$$

where, $0<k \leq \frac{1}{8}$ and $\phi: \mathbb{R}_{+} \rightarrow \mathbb{R}_{+}$is such that $\phi(t)<t$ for each $t>0$. If one of the mappings $A, B$, or $I_{X}$ be continuous then $A, B$ and $I_{X}$ have a unique common fixed point.

Remarks: Our results extend and improve the results of Meir-Keeler [3], Bouhadjera and Djoudi [24], Jha, Pant and Singh [25], Pant and Jha [26] in dislocated metric space.

\section{Acknowledgements}

This work is carried out under Small Research Development and Innovation Grant SRDIG-73/74-S\&T-08 supported by University Grants Commission, Nepal.

\section{References}

[1] Brouwer, L.E.J. (1912) Uber Abbildung von Mannigfaltigkeiten. Mathematische Annalen, 71, 97-115. https://doi.org/10.1007/BF01456931

[2] Banach, S. (1922) Sur les operations dans les ensembles abstraits et leur applications aux equations integrals. Fundamental Mathematicae, 3, 133-181.

[3] Meir, A. and Keeler, E. (1969) A Theorem in Contraction Mappings. Journal of Mathematical Analysis and Applications, 28, 326-329. https://doi.org/10.1016/0022-247X(69)90031-6

[4] Maiti, M. and Pal, T.K. (1978) Generalization of Two Fixed-Point Theorems. Bulletin of the Calcutta Mathematical Society, 70, 57-61.

[5] Park, S. and Rhoades, B.E. (1981) Meir-Keeler Type Contractive Conditions. Mathematica Japonica, 26, 13-20.

[6] Rao, I.H.N. and Rao, K.P.R. (1985) Generalization of Fixed-Point Theorems of Meir-Keleer Type. Indian Journal of Pure and Applied Mathematics, 16, 1249-1262.

[7] Jungck, G. (1986) Compatible Mappings and Common Fixed Points. International Journal of Mathematics and Mathematical Sciences, 9, 771-779. https://doi.org/10.1155/S0161171286000935

[8] Pant, R.P. (1986) Common Fixed Point of Two Pair of Commuting Mappings. Indian Journal of Pure and Applied Mathematics, 17, 187-192.

[9] Carbone, A., Rhoades, B.E. and Singh, S.P. (1989) A Fixed Point Theorem for Ge- 
neralized Contraction Map. Indian Journal of Pure and Applied Mathematics, 20, 543-548.

[10] Matkowski, J. (1975) Integrable Solutions of Functional Equations. Dissertationes Mathematicae, 127.

[11] Jachymski, J. (1994) Common Fixed Point Theorems for Some Families of Mappings. Indian Journal of Pure and Applied Mathematics, 25, 925-937.

[12] Pant, R.P. (1998) Common Fixed Points of Contractive Maps. Journal of Mathematical Analysis and Applications, 226, 251-258.

https://doi.org/10.1006/jmaa.1998.6029

[13] Boyd, D.W. and Wong, J.S. (1969) On Nonlinear Contractions. Proceedings of the American Mathematical Society, 20, 458-464. https://doi.org/10.1090/S0002-9939-1969-0239559-9

[14] Park, S. and Rhoades, B.E. (1981) Extension of Some Fixed Point Theorems of Hegedus and Kasahara. Mathematics Seminar Notes, 9, 113-118.

[15] Matthews, S.G.(1986) Metric Domains for Completeness. Technical Report 76, Ph.D. Thesis, University of Warwick, Coventry.

[16] Hitzler, P. and Seda, A.K. (2000) Dislocated Topologies. Journal of Electrical Engineering, 51, 3-7.

[17] Kumari, P.S., Zoto, K. and Panthi, D. (2015) $d$-Neighborhood System and Generalized F-Contraction in Dislocated Metric Space. SpringerPlus, 4, 368. https://doi.org/10.1186/s40064-015-1095-3

[18] Kumari, P.S., Ramana, C.V., Zoto, K. and Panthi, D. (2015) Fixed Point Theorems and Generalizations of Dislocated Metric Spaces. Indian Journal of Science and Technology, 8, 154-158. https://doi.org/10.17485/ijst/2015/v8iS3/62247

[19] Panthi, D. (2015) Common Fixed Point Theorems for Compatible Mappings in Dislocated Metric Space. International Journal of Mathematical Analysis, 9, 2235-2242. https://doi.org/10.12988/ijma.2015.57177

[20] Panthi, D. and Kumari, P.S. (2016) Some Integral Type Fixed Point Theorems in Dislocated Metric Space. American Journal of Computational Mathematics, 6, 88-97. https://doi.org/10.4236/ajcm.2016.62010

[21] Panthi, D. and Subedi, K. (2016) Some Common Fixed Point Theorems for Four Mappings in Dislocated Metric Space. Advances in Pure Mathematics, 6, 695-712. https://doi.org/10.4236/apm.2016.610057

[22] Jungck, G. and Rhoades, B.E. (1998) Fixed Points for Set Valued Functions without Continuity. Indian Journal of Pure and Applied Mathematics, 29, 227-238.

[23] Pant, R.P. (1999) A Common Fixed Point Theorem under a New Condition. Indian Journal of Pure and Applied Mathematics, 30, 147-152.

[24] Bouhadjera, H. and Djoudi, A. (2008) On Common Fixed Points of Meir and Keeler Type. Analele Stiintifice ale Universitatii Ovidius Constanta, 16, 39-46.

[25] Jha, K., Pant, R.P. and Singh, S.L. (2003) Common Fixed Points for Compatible Mappings in Metric Space. Radovi Matematicki, 12, 107-114.

[26] Pant, R.P. and Jha, K. (2002) A Generalization of Meir-Keeler Type Common Fixed Point Theorem for Four Mappings. Journal of Natural and Physical Sciences, 16, 77-84. 